\title{
Recurrent Myocarditis Imitating ST Segment Elevation Myocardial Infarction
}

\author{
Anthony E. Peters ${ }^{\mathrm{a}}$, Adrian Loffler ${ }^{\mathrm{b}, \mathrm{c}}$, Christopher M. Kramer ${ }^{\mathrm{b}, \mathrm{c}}$, \\ Michael Salerno ${ }^{\text {b, c, d }}$, Younghoon Kwon ${ }^{\text {, e }}$
}

\begin{abstract}
Acute myocarditis mimicking ST segment elevation myocardial infarction is a well-known phenomenon, but recurrence and progression are rare. We present a case of a 29-year-old man with three episodes of myocarditis over 3 months including evidence of progressive, patchy inflammation shown by cardiovascular magnetic resonance imaging.
\end{abstract}

Keywords: Myocarditis; Late gadolinium enhancement; T1 and T2 mapping; Cardiovascular magnetic resonance imaging

\section{Introduction}

Myocarditis imitating acute myocardial infarction (MI) is welldescribed in young patients without major cardiac risk factors. However, recurrence is uncommon, and typically occurs "late", several months to years after initial presentation [1-7]. Multiple, rapid recurrences over the course of weeks to months are particularly rare [8]. This case represents one of the few documented reports of recurrent myocarditis over a short time period. Cardiovascular magnetic resonance imaging (CMR) was critical to the diagnosis in this case as it demonstrated multi-focal late gadolinium enhancement including characteristic epicardial foci.

\section{Case Report}

A 29-year-old obese, but otherwise healthy, African American

Manuscript submitted May 7, 2018, accepted May 25, 2018

aDepartment of Medicine, University of Virginia Health System, Charlottesville, VA, USA

bDepartment of Medicine, Cardiology Division, University of Virginia Health System, Charlottesville, VA, USA

'Department of Radiology, Cardiovascular Imaging Center, University of Virginia Health System, Charlottesville, VA, USA

dDepartment of Biomedical Engineering, University of Virginia Health System, Charlottesville, VA, USA

${ }^{\mathrm{e}}$ Corresponding Author: Younghoon Kwon, Division of Cardiovascular Medicine, University of Virginia, PO Box 800158, Charlottesville, VA 22908-0158, USA.Email: yk2j@virginia.edu

doi: https://doi.org/10.14740/jmc3081w male without history of medication use or substance abuse presented to the emergency room with sudden onset substernal chest pain at rest, accompanied by vomiting, left arm weakness, and fatigue. One week prior to admission he had transient upper respiratory symptoms. Electrocardiogram (ECG) showed diffuse ST-elevations, most prominent in the inferior leads. Troponin I was elevated to $8.45 \mathrm{ng} / \mathrm{mL}$. An echocardiogram was unremarkable with only a trivial pericardial effusion. Given concern for myocarditis, CMR was obtained for diagnosis and prognostication. This showed patchy late gadolinium enhancement (LGE) in the epicardium of the mid-toapical inferior walls consistent with myocarditis (Fig. 1A, B). He was treated with nonsteroidal anti-inflammatory medication (NSAID) as needed and colchicine as he was initially felt to have a component of pericarditis.

After 2 months without symptoms, the patient presented again with chest pain, similar to the prior presentation, albeit more intense. ECG showed $3 \mathrm{~mm}$ ST segment elevations in the inferior leads and $1 \mathrm{~mm}$ in the lateral leads with no appreciable PR depression, concerning for an inferior ST elevation myocardial infarction (STEMI) (Fig. 2A, B). Troponin peaked at 39.5. His ECG evolved overnight with the development of Q waves and T wave inversions (Fig. 2A, B). There was a new inferior wall motion abnormality on echocardiogram. Due to concern for possible STEMI, cardiac catheterization was performed, revealing normal coronary arteries without evidence of thrombus or vasospasm (Fig. 2). Repeat CMR showed inferior and anteroseptal hypokinesis and evidence of focal areas of progressive LGE in the mid-basal inferior walls, along with increased T1 and T2 values consistent with acute myocarditis (Fig. 3A, B). There was patchy-like multi-focal LGE involving the basal inferior wall, distal inferior wall, and septum with regions of epicardial LGE, supporting the diagnosis of myocarditis. Telemetry demonstrated no cardiac conduction abnormalities during his hospitalization. He was discharged on an angiotensin-converting enzyme (ACE) inhibitor and beta blocker. His symptoms soon resolved. At a follow-up clinic visit, his ECG showed large inferior Q-waves.

One month later, the patient presented again with acute substernal chest pain during exertion. He manifested ECG changes with diffuse ST elevations, troponin I elevation, peaking at 10.6, and follow-up CMR findings of focal inferior LGE, now nearly transmural. Comprehensive rheumatological and infectious workup including autoimmune (antinuclear antibody, rheumatoid factor, cyclic citrullinated peptide IgG, antismooth muscle antibody, liver-kidney microsomal antibody, 


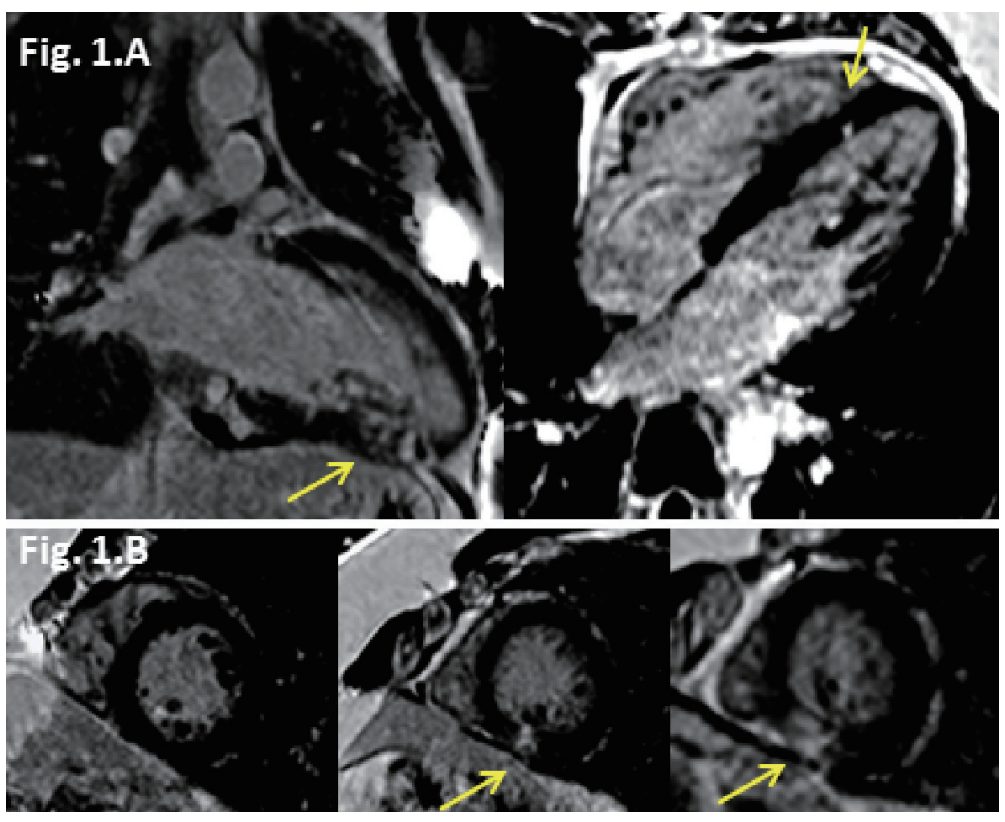

Figure 1. Late gadolinium enhancement (LGE) (yellow arrows) cardiovascular magnetic resonance (CMR) images from first admission. (A) Two chamber view showing mid and epicardial LGE along the inferior wall, and 4 chamber view showing epicardial LGE along the mid inferoseptal wall. (B) Short axis views (basal to apical) showing mid and epicardial LGE along the inferior wall.

angiotensin converting enzyme, quantitative immunoglobulins), inflammatory (sedimentation rate, C-reactive protein), and infectious (HIV, hepatitis serologies, Lyme serology, cytomegalovirus antibody) etiologies were unrevealing.

Over the following months, the patient was followed closely in cardiology and primary care clinics and he has done reasonably well. At his most recent visit (18 months after his initial presentation), the patient had started exercising again and had no symptoms related to his myocarditis. His most recent CMR demonstrated mild dilatation of the left ventricle with normal ejection fraction, some unchanged patchy foci of LGE, and a segmental wall motion abnormality at the inferior

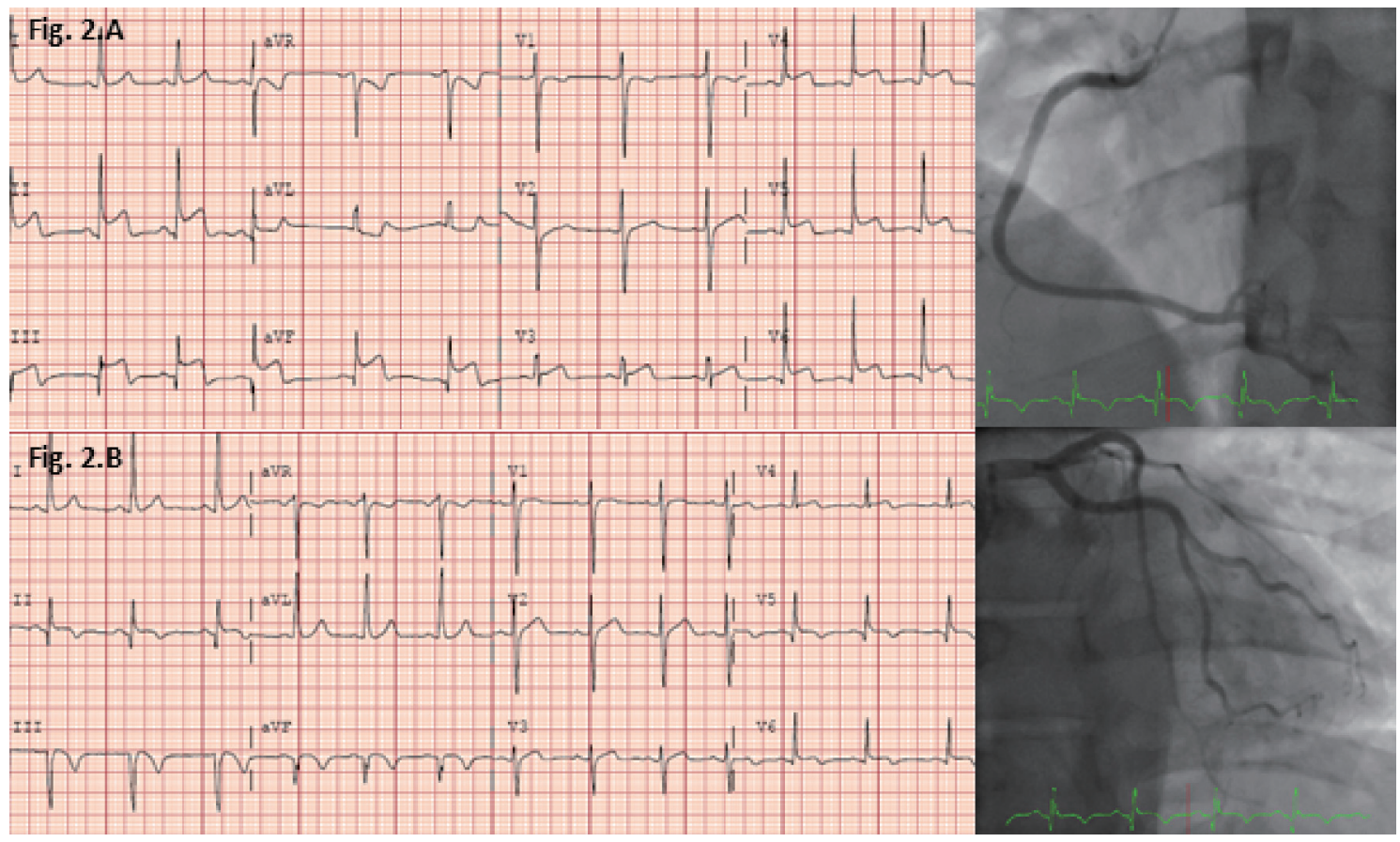

Figure 2. Chronological ECGs (day 1 at 1:01 PM, day 2 at 1:33 AM) during the second admission and cardiac catheterization during the second admission. 


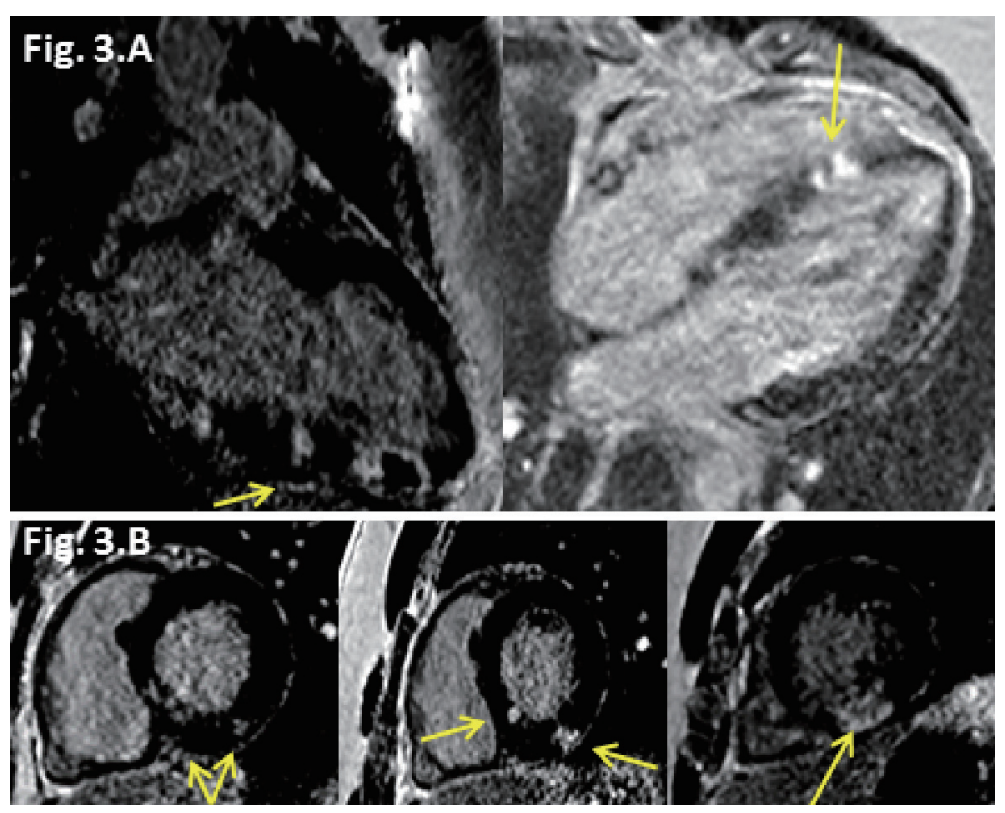

Figure 3. Late gadolinium enhancement (LGE) (yellow arrows) cardiovascular magnetic resonance (CMR) images from the second admission. (A) Two chamber view showing progression of LGE (transmural) along the inferior wall, and 4 chamber view showing progression of LGE (transmural) along the mid inferoseptal wall. (B) Short axis views (basal to apical) showing new patchy epicardial to mid LGE in inferolateral and inferoseptal region walls.

and inferolateral mid to apical segments with associated transmural LGE.

\section{Discussion}

In this case, each of the three clinical presentations represented a distinct episode of acute recurrent myocarditis as evidenced by acute development and resolution of symptoms, evolution of ECG findings, trends of cardiac injury biomarker, and signs of acute inflammation, albeit overall progression, by CMR for each presentation. This recurrent pattern over a short period of time (three events over 3 months) posed clinical challenges in assuring the diagnosis. Despite the established diagnosis of myocarditis during the first presentation, the second presentation with similar but more dramatic symptoms and ECG changes caused concern for potential de novo acute MI. Indeed, the focal nature of pathology with inferior ECG changes and corresponding wall motion abnormality closely mimicked STEMI and ultimately led to pursuit of coronary angiography. While angiography remains indicated in these clinical scenarios, this case underscores the potential for recurrent, severe myocarditis that can mimic acute coronary syndrome.

CMR was critical in diagnosis and characterizing the evolving pathology. This diagnosis was supported by the progression of multi-focal LGE and edema along the inferior wall, as well as foci of epicardial LGE in a pattern typical of myocarditis (mid and epicardial wall, sparing endocardium) along with demonstration of completely normal coronary arteries on angiography. Further, the pattern of increased T1 and T2 mapping provided evidence of myocardial edema and was indicative of acute myocarditis. This imaging alone was not conclusive for myocarditis and the differential diagnosis also included a vasospastic process. However, the combination of the patient's symptoms and lack of risk factors for coronary disease along with the CMR findings of patchy epicardial LGE recurring in the same anatomical location made recurrent myocarditis the most likely explanation for this patient's presentations.

The etiology of this patient's recurrent myocarditis remains unclear. Known etiologies of myocarditis include infectious (group A streptococcus, influenza, and other viral infections), autoimmune-mediated (lupus, sarcoidosis, lymphocytic, and giant-cell), malignancy-associated (diffuse large B-cell lymphoma), and drug-associated (hypersensitivity and toxic) causes $[2-5,7,8]$. In this case, workup remained unrevealing, leaving only primary viral illness (albeit mild), re-infection, and/or un-identified post-infectious autoimmune syndrome as potential causes. Endomyocardial biopsy was deferred given the particular challenges in this patient with a focal lesion in the inferior left ventricle without involvement of the right ventricle. In consultation with rheumatology, it was determined that anti-inflammatory and immunomodulatory agents such as corticosteroids or intravenous immunoglobulin were not indicated. Since his third admission, the patient has been continued on ACE inhibitor and beta blocker and has been advised to avoid NSAIDs, alcohol, and any exertional physical activity for the next 6 months.

The course of myocarditis varies by etiology and the severity of presenting symptoms. In a small portion of patients with acute myocarditis, ventricular dysfunction and arrhythmias or conduction disturbances may persist for months to years owing to scar or rarely, as illustrated in our case, persistent or recurrent active myocarditis. 
This report highlights an unusual case of recurrent myocarditis mimicking acute MI and demonstrates CMR as an important tool for diagnosing, monitoring, and potentially prognosticating the progression of recurrent myocarditis.

\section{Financial Support}

This work was supported by NIH R01 grant HL131919-01A1 to Salerno.

\section{References}

1. Costantini M, Oreto G, Albanese A, Ranieri A, De Fabrizio G, Sticchi I, Lauretti A, et al. Presumptive myocarditis with ST-Elevation myocardial infarction presentation in young males as a new syndrome. Clinical significance and long term follow up. Cardiovasc Ultrasound. 2011;9:1.

2. Varnavas VC, Reinsch N, Perrey M, Nensa F, Schlosser T, Baba HA, Gerken G, et al. Recurrent lymphocytic myocarditis in a young male with ulcerative colitis. Eur J Med Res. 2014;19:11.
3. Xu B, Michael Jelinek V, Hare JL, Russell PA, Prior DL. Recurrent myocarditis - an important mimic of ischaemic myocardial infarction. Heart Lung Circ. 2013;22(7):517522.

4. Lee SH, Choi SA, Choi JH, Kim CW, Shin HJ, Oh JH. Recurrent fulminant myocarditis associated with diffuse large B-cell lymphoma. Int J Cardiol. 2013;164(1):e7-8.

5. Maleszewski JJ, Orellana VM, Hodge DO, Kuhl U, Schultheiss HP, Cooper LT. Long-term risk of recurrence, morbidity and mortality in giant cell myocarditis. Am J Cardiol. 2015;115(12):1733-1738.

6. Kanazawa H, Hata N, Yamamoto E, Shinada T, Yokoyama $\mathrm{S}$, Ohba T, Imaizumi T, et al. Recurrent myocarditis of unknown etiology. J Nippon Med Sch. 2004;71(4):292296.

7. Karavidas A, Lazaros G, Noutsias M, Matzaraki V, Danias PG, Pyrgakis V, Voudris V, et al. Recurrent coxsackie $\mathrm{B}$ viral myocarditis leading to progressive impairment of left ventricular function over 8 years. Int J Cardiol. 2011;151(2):e65-67.

8. Takehana H, Inomata T, Kuwao S, Nakahata J, Sasaki T, Nishii M, Kurokawa S, et al. Recurrent fulminant viral myocarditis with a short clinical course. Circ J. 2003;67(7):646-648. 